\title{
Sensing underground coal gasification by ground penetrating radar
}

\author{
Andrzej Kotyrba ${ }^{1}$ (D) Krzysztof Stańczyk $^{2}$
}

Received: 3 March 2017/Accepted: 1 November 2017/Published online: 13 November 2017

(c) The Author(s) 2017. This article is an open access publication

\begin{abstract}
The paper describes the results of research on the applicability of the ground penetrating radar (GPR) method for remote sensing and monitoring of the underground coal gasification (UCG) processes. The gasification of coal in a bed entails various technological problems and poses risks to the environment. Therefore, in parallel with research on coal gasification technologies, it is necessary to develop techniques for remote sensing of the process environment. One such technique may be the radar method, which allows imaging of regions of mass loss (voids, fissures) in coal during and after carrying out a gasification process in the bed. The paper describes two research experiments. The first one was carried out on a large-scale model constructed on the surface. It simulated a coal seam in natural geological conditions. A second experiment was performed in a shallow coal deposit maintained in a disused mine and kept accessible for research purposes. Tests performed in the laboratory and in situ conditions showed that the method provides valuable data for assessing and monitoring gasification surfaces in the UCG processes. The advantage of the GPR method is its high resolution and the possibility of determining the spatial shape of various zones and forms created in the coal by the gasification process.
\end{abstract}

Andrzej Kotyrba

a.kotyrba@gig.eu

Krzysztof Stańczyk

k.stanczyk@gig.eu

1 Department of Geology and Geophysics, Central Mining Institute, Katowice, Poland

2 Department of Energy and Air Protection, Central Mining Institute, Katowice, Poland
Keywords GPR · Coal · Gasification · Sensing

\section{Introduction}

In recent times, the traditional combustion of fossil fuels, and particularly coal, has acquired a bad reputation due to air pollution and its impact on climate (Gaffney and Marley 2009; Shindell and Faluvegi 2010). Nevertheless, the widespread occurrence of coal resources means that coal, at present and most probably in the future, will continue to be used as an energy source. If the energy could be extracted from the coal without transportation to the surface, the process would have less impact on the environment. This is the most promising effect of underground coal gasification (UCG) technology which allows energy to be obtained in the form of gas discharged to the surface as a result of the in situ coal seam gasification. The obtained gas is a product of industrial value that can be used in the production of electricity and heat or as a synthesis gas. The UCG technologies are the subject of many publications. The technical solutions applied depend on geological conditions of coal basins, type of coal beds, physical and chemical properties of coal and other factors as well (Bhutto et al. 2013; Kostur et al. 2015).

Regardless of the underground coal gasification technology, the process poses various risks to the environment that have to be controlled remotely. Among various geophysical methods, one such control technique may be the ground penetrating radar (GPR) method. This method illuminates the gasification area in the coal with electromagnetic pulses at microwave frequencies and records the signals reflected by its borders (Turner, Yelf and Hetherly 1989). The high transmit rate of GPR systems and the frequency of radar pulses $(25-2000 \mathrm{MHz})$ provide spatial 
resolution quality that is unattainable by other geophysical methods. However, the disadvantage of the method is its relatively low penetration range in the geological environment due to water bonded in the structure of the rocks and minerals. Water has a dominant impact on the attenuation of electromagnetic signals in rocks (Annan 2001). The process of underground coal gasification (UCG), due to its high temperature, transforms the water from liquid phase to gaseous phase. The gasification process also changes the coal structure. Around the gasification zone, this creates a space with fissures that enable the process to progress (Itakura et al. 2009; Su et al. 2013; Bhutto et al. 2013). For determination of the shape and size of gasification cavity as well as progress of the process in time domain, various methods are used. Among them are numerical tools which use out solutions of computational fluid dynamics (CFD) for modelling and simulation of gasification process (Nourozieh H et al. 2010; Janoszek et al. 2013; Sarraf et al. 2013). A comprehensive review of underground coal gasification modelling works was recently done by Khan et al. (2015).

The propagation of electromagnetic waves in rocky materials depends greatly on their thermo-electrical properties. The first trials to apply the radar method for imaging of gasification voids were conducted within the RFCS-funded project (acronym HUGE) on a large-scale model simulating underground conditions (Stańczyk et al. 2010). Collected radar data were processed with 2D algorithms (Kotyrba and Stańczyk 2013). This procedure enabled two reflecting interfaces to be determined within the coal, corresponding to gasification and cavity surfaces. The cavity was visible clearly on the data recorded after process termination, but rather badly on the data recorded during the gasification stage. In datasets collected during the gasification stage, the interface of the gasification zone was the dominant reflector of electromagnetic pulses in the amplitude domain. Both interfaces had curved trends. This paper describes the results of two GPR surveys conducted on a model and during inseam underground coal gasification with the application of 3D methods for data visualization.

\section{Propagation of electromagnetic waves in coal and gasification products}

According to the theory of electromagnetics, the basic parameters that govern and describe the process of electromagnetic waves propagation, reflection and transmission in material media are determined by Eqs. 1, 2, 3 and 4 (Crawford 1968; Annan 2001).

$Z=\frac{E}{H}$ where $Z$ is the electromagnetic impedance of medium (complex number), $E$ is the intensity of electric field (vector) and $H$ is the intensity of magnetic field (vector).

Electromagnetic impedance $Z$ (ohm) is a complex number that depends on the electromagnetic properties of the medium and the frequency of the propagating electromagnetic field.

$Z=\sqrt{\frac{\mu}{\varepsilon+j \frac{\sigma}{\omega}}}$

where $\mu$ is the magnetic permeability, $\varepsilon$ is the electric permittivity, $\sigma$ is the electrical conductivity $(\mathrm{S} / \mathrm{m})$ - reciprocal of electrical resistivity $\rho$ (ohm-m), $\omega$ is the angular frequency of electromagnetic field $(2 \pi f)$, and $f$ is the frequency of electromagnetic field $(\mathrm{Hz})$.

In practical GPR applications, the magnetic properties of media have no influence on propagation (coal like many other rocks has paramagnetic properties and its relative magnetic permeability is equal to 1 ). Therefore, the magnetic permeability is usually omitted in equations. The energy of an electromagnetic field propagating in a medium is attenuated due to scattering, spherical divergence, energy partitioning at interfaces and absorption. The absorption is low in weakly conductive media and high in strongly conductive media.

Dielectric permittivity is a complex number in which imaginary part $\varepsilon$ " describes the polarization ability of the medium according to Eq. (3):

$\varepsilon=\varepsilon+i \varepsilon^{\prime \prime}=\varepsilon^{\prime}(1+i \tan \delta)$

where $\varepsilon^{\prime}$ is real part of dielectric permittivity, $\varepsilon^{\prime \prime}$ is the imaginary part of dielectric permittivity and $\tan \delta$ is the dissipation factor (due to polarization and heat).The amplitude of the radar wave reflected by an interface is determined by reflection coefficient $R$ which can be expressed by Eq. (4):

$R=\frac{Z_{2}-Z_{1}}{Z_{2}+Z_{1}}$

where $Z_{1}$ (ohm) is the electric impedance of the part of the medium from which the wave travels to the reflecting interface. The part of the medium behind the reflecting interface has the electric impedance $Z_{2}$.

For highly electrically resistive media (such as rocks and in particular burning coal) (Hanninen et al. 1992), the reflection coefficient can be expressed without taking into account the conductivity from Eq. (5):

$R=\frac{\sqrt{\varepsilon_{1}}-\sqrt{\varepsilon_{2}}}{\sqrt{\varepsilon_{1}}+\sqrt{\varepsilon_{2}}}$

where $\varepsilon_{1}$ and $\varepsilon_{2}$ are the dielectric constants of the two materials at the interface. For a standard GPR antenna 
transducers frequency bandwidth attenuation of radar signals in rock mass, in simplified form can be expressed by Eq. (6) (Hanninen et al. 1992):

$A=\frac{1635 \sigma}{\sqrt{\varepsilon}}=\frac{1635}{\rho \sqrt{\varepsilon}}$

where $A$ is the attenuation $(\mathrm{dB}), \sigma$ is the electric conductivity $(\mathrm{S} / \mathrm{m})$ and $\rho$ is the electric resistivity (ohm-m).

According to laboratory investigations, the electrical resistivity of coal depends strongly on temperature. For the temperature range $20-1900{ }^{\circ} \mathrm{C}$, the relation can be approximated by a polynomial of fourth order (Kotyrba 1999) and relation (6) can be expressed as:

$A(T)=\frac{1635}{\rho_{o}\left(c_{1} T^{4}-c_{2} T^{3}+c_{3} T^{2}-c_{4} T+c_{5}\right) \sqrt{\varepsilon}}$

where $\rho_{0}$ is the resistivity at $20{ }^{\circ} \mathrm{C}(\mathrm{ohm}-\mathrm{m}), T$ is the temperature $\left({ }^{0} \mathrm{C}\right)$ and $c_{1}, c_{2}, c_{3}, c_{4}, c_{5}$ are the coefficients depending on the coal type, rank and moisture (to be determined from data obtained in laboratory oven tests).

For a milled and washed coal sample from the Polish Janina mine, characterized by resistivity value $1100 \mathrm{O}-\mathrm{m}$ at $20{ }^{\circ} \mathrm{C}$ and considering four selected dielectric constants values (from a variation range observed in Polish hard coals), the relation between attenuation and temperature within the range $20-1900{ }^{\circ} \mathrm{C}$ calculated from Eq. (7) is shown in Fig. 1. According to Eq. (4), the amplitude of electromagnetic wave reflection depends on the difference of complex impedances on the interfaces created in the coal by the gasification process (coal-char, coal-gas, chargas) or only from values of dielectric permittivity, if we use Eq. (5). The term char is used here for a partially degassed coal.

Tests conducted on the electric properties of flame during gas combustion have shown that the dielectric permittivity of the flame increases only by $4 \%$ in relation to

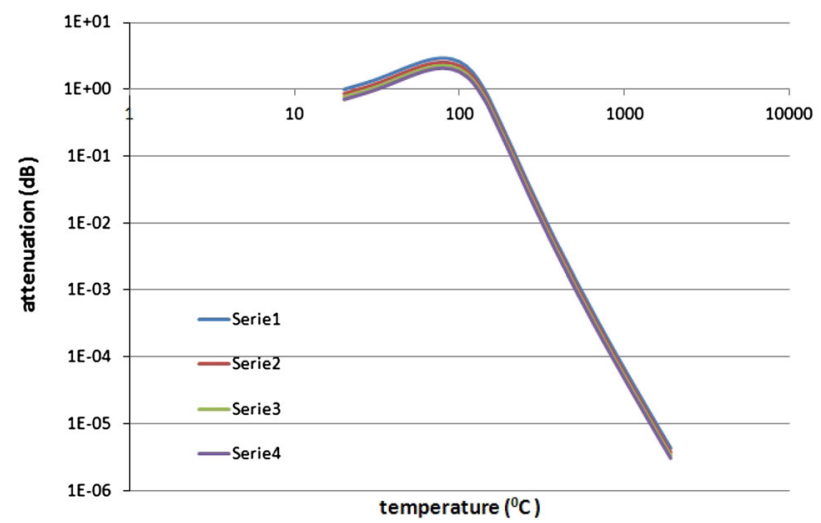

Fig. 1 Attenuation of electromagnetic wave versus temperature plotted for selected coal sample $\left(\rho_{0}=1100 \mathrm{O}-\mathrm{m}\right)$ in temperature range $20-1900{ }^{\circ} \mathrm{C}(\varepsilon=3$ serie $1, \varepsilon=4$ serie $2, \varepsilon=5$ serie $3, \varepsilon=6$ serie4) source gas (Gut et al. 2004). This allows us to omit the polarization effect in the combustion zone (present due to separation of charges on the combustion surface during temperature rise in the flame). The energy of reflections depends mainly on the spatial distribution of the variously transformed (carbonized or burnt out) parts of the coal mass (zones characterized by different permittivity and conductivity values), when we compare images recorded before and after the gasification process. Nevertheless, the polarization phenomena cannot be omitted when comparing radar data recorded during gasification. The flow of the gases mixture with unbalanced charges along the surfaces created between the coal and char and within the char (fissures) causes the out-of-phase additional current and an increase of the imaginary part of the dielectric constant. According to Eq. (3), this also causes a change in the effective dielectric permittivity of coal within the gasification zone (Kotyrba and Stańczyk 2013).

In the temperature range from app. $100-1000{ }^{\circ} \mathrm{C}$, the electrical resistivity of the coal increases from an order of approximate $10^{2}$ to $10^{6}$ O-m (Kotyrba 1999).

Therefore, from Eqs. (6) and (7), it can be estimated that when the coal has a temperature of several hundred degrees Celsius, the attenuation of waves in the seam rapidly drops. This can significantly increase the penetration range of electromagnetic pulses in real geologic conditions and the overall penetration performance of radar systems.

In the gasification process, water is also released from the coal due to its natural moisture. Depending on internal pressure up to a critical point at $T=374{ }^{\circ} \mathrm{C}$ and $p=220$ bars, the water can occur in liquid and gaseous states, but at temperatures exceeding critical point, the water can occur only in gaseous state (Himmelblau 1960; Ebbing and Gammon 2011). In the gasification zone, the pressure of gases does not exceed 1-3 bars. Taking into account that in the process of hard coal gasification, temperatures range from a dozen to $1500{ }^{\circ} \mathrm{C}$ so it can be assumed that when the temperature in gasification zone exceeds the boiling point $\left(\sim 100^{\circ} \mathrm{C}\right)$, most of water is transformed to vapour.

Reverse transition occurs during the extinction of georeactor by cooling (injection of nitrogen to gasification zone). Therefore, it can be assumed that during the coal gasification process (comprising a heating and cooling phase) the water can affect radar waves propagation in early and late phases of process. This was confirmed in research tests carried out on British coals (Marland et al. 2001).

Water has a major impact on radar images while it is in liquid state. The value of the dielectric constant of water is very high compared to coal and its gasification products. It depends on the temperature of the water. When the water transforms to gaseous state (vapour), the value of the dielectric constant decreases to the level of other gaseous products of coal gasification (Table 1). 
Table 1 Dielectric properties of coal and gasification products

\begin{tabular}{lllll}
\hline Medium & Temperature $\left({ }^{\circ} \mathrm{C}\right)$ & Temperature $\left({ }^{\circ} \mathrm{F}\right)$ & Dielectric constant & Phase state \\
\hline Coal (hard) & 20 & 68.0 & $2.5-5.0^{\mathrm{a}}$ & Solid \\
Coke (char) & Undefined & Undefined & $1.1-2.2^{\mathrm{b}}$ & Solid \\
Carbon black & Undefined & Undefined & $2.5-3.0^{\mathrm{b}}$ & Solid \\
Coal tar & Undefined & Undefined & $2.0-3.0^{\mathrm{b}}$ & Solid \\
Coal powder (fine) & Undefined & Undefined & $4^{\mathrm{b}}$ & Solid \\
Fly ash & 20 & 68.0 & $1.7-2.2^{\mathrm{b}}$ & Solid \\
Hydrogen & 100 & 212 & $1.000284^{\mathrm{b}}$ & Gaseous \\
Hydrogen & 226.7 & 440 & $1.23^{\mathrm{b}}$ & Gaseous \\
Oxygen & 29.3 & 84.7 & $1.0004829^{\mathrm{c}}$ & Gaseous \\
Methane & 29.3 & 84.7 & $1.0008029^{\mathrm{c}}$ & Gaseous \\
Carbon dioxide & 29.3 & 84.7 & $1.0064989^{\mathrm{c}}$ & Gaseous \\
Nitrogen & 29.3 & 84.7 & $1.0080294^{\mathrm{c}}$ & Gaseous \\
Water steam & 20 & 68 & $1.000785^{\mathrm{b}}$ & Gaseous \\
Water & 0 & 32 & $88.0^{\mathrm{b}}$ & Liquid \\
Water & 20 & 68 & $80.4^{\mathrm{b}}$ & Liquid \\
Water & 100 & 212 & $55.3^{\mathrm{b}}$ & liquid \\
\hline
\end{tabular}

${ }^{a}$ Kotyrba \& Stańczyk (Kotyrba and Stanczyk 2013)

${ }^{\text {b }}$ Raumenergie-Förder Gesellschaft (2013)

${ }^{c}$ Schmidt and Moldover (2003)
When the gasification process is terminated, one might expect that the strongest reflections should come from interfaces between reacted (char) and non-transformed coal and from the surfaces of voids (cavities and fissures). Computation of the reflection coefficient on coal-char and char-gas interfaces from formula 5 for selected values in the variation range from Table 1 give us the values listed in Table 2.

The reflection coefficients depend strongly on the dielectric constants of coal and char. In cases 1 and 2 (where mean values of dielectric constant are taken), they are different for both interfaces. This can make it possible to distinguish both faces in the GPR data sets. In cases 3 and 4 , both interfaces have similar reflection coefficients. Therefore, it may be impossible to distinguish them from one another in the data. The highest reflection coefficient occurs at the gas-water interface (case 5). This product can have a significantly greater impact on the radar images due to its relatively high dielectric permittivity value (Table 1). It should be noted that on the interface gas-water also the phase of radar signal changes the sign $(\mp)$.

\section{GPR survey on a laboratory model}

The first GPR experiments were carried out on a laboratory model. In primary theoretical considerations, we assumed that it can be described by the two-layer medium consisting of brick wall and coal with gasification void. The conceptual scheme of GPR measurement (reflection mode) for void detection and control of its growth are shown in Fig. 2.

A real "ex situ" model was constructed as a rectangular structure with brick walls and set on a foundation frame. Large coal blocks of approximately $2 \mathrm{~m}$ long and 0.8-1.2 $\mathrm{m}$ wide were placed inside the structure. After placing the coal specimen in the structure, the free space between the walls and block faces was filled with sand. Sand was also used to fill the space between the top face of the coal specimen and the metal plate closing the construction at the top. The brick construction of the model was adjusted to conduct experiments on various coal types and configurations of flue channels. In the experiment described in this paper, V-shaped flue channels were used.

The vertical section of the model and the location of a set of 22 measurement profiles on one of its lateral faces are shown in Fig. 3. The spacing between profiles was set to $10 \mathrm{~cm}$.

The horizontal section of the model showing the orientation of the flue channels is depicted in Fig. 4.

The process of gasification started with ignition of the coal. Afterwards, oxygen was supplied to the coal through an inlet hole, followed by extraction of process gases via the outlet hole. On the model, two series of radar measurements were carried on. The SIR 3000 radar system equipped with a $1000 \mathrm{MHz}$ standard transducer was used. A photo views of measurement on "ex situ" model and the GPR equipment used in "ex situ" and "in situ" conditions are shown in Fig. 5a, b. 
Table 2 Reflection coefficients on gasification interfaces

\begin{tabular}{lllcc}
\hline Case & Interface & $\varepsilon_{1}$ & $\varepsilon_{2}$ & Reflection coefficient \\
\hline 1 & Coal-char & 3.75 & 1.65 & 0.20 \\
2 & Char-gas & 1.65 & 1.00 & 0.12 \\
3 & Coal-char & 5.00 & 2.20 & 0.20 \\
4 & Char-gas & 2.20 & 1.00 & 0.19 \\
5 & Gas-water (at $\left.T=100^{\circ} \mathrm{C}\right)$ & 1.00 & 55.3 & -0.76 \\
\hline
\end{tabular}

Fig. 2 Scheme of measurement array and the travel paths of radar signals in a laboratory model with void ( $T$ transmitter, $R$ receiver, scan amplitude versus time record of single travel path, $V$ velocity of radar wave, $\varepsilon$ dielectric constant, $\rho$ specific electrical resistivity)
Fig. 3 Layout of GPR profiles on vertical face of the laboratory model (side view)
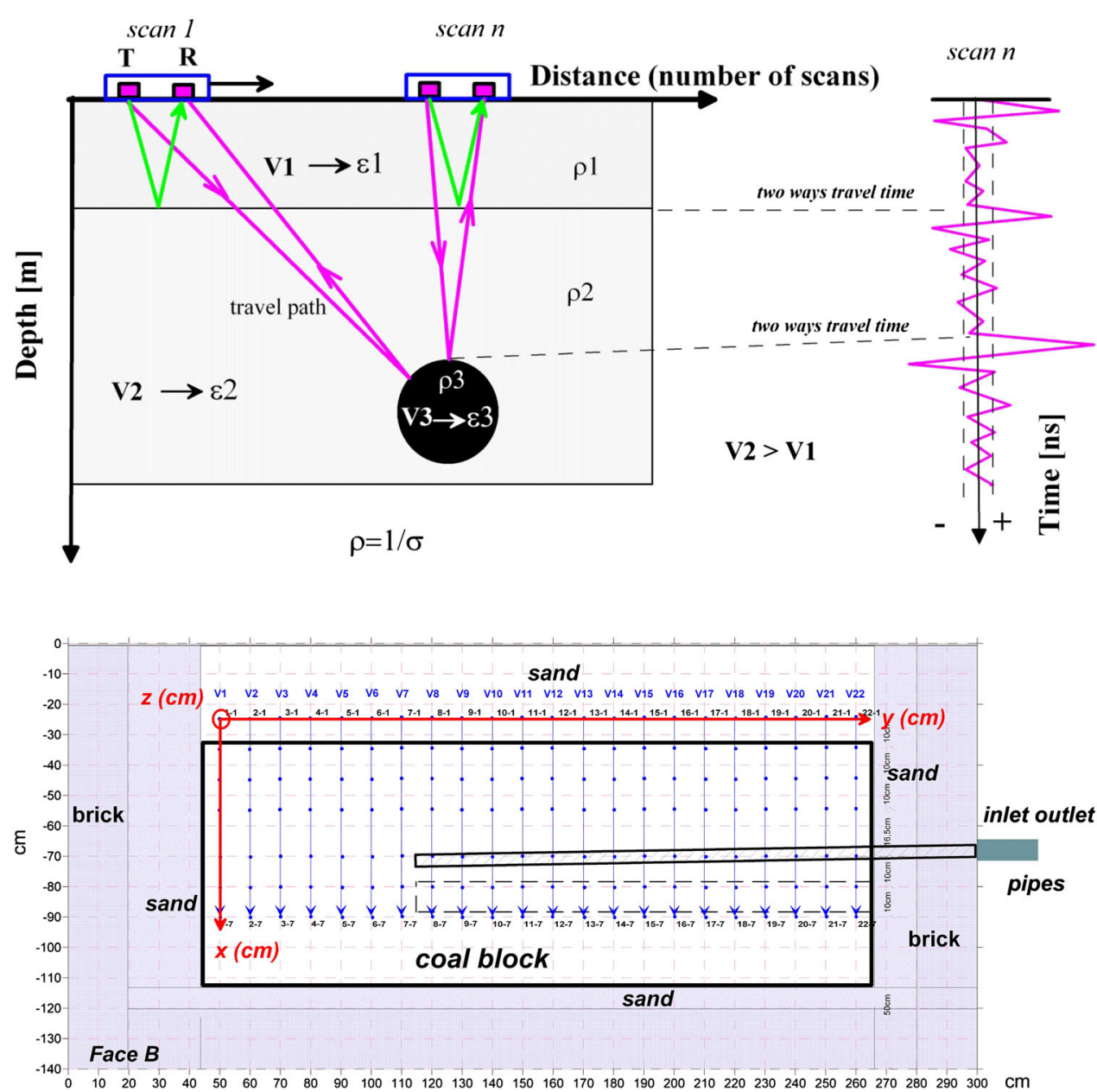

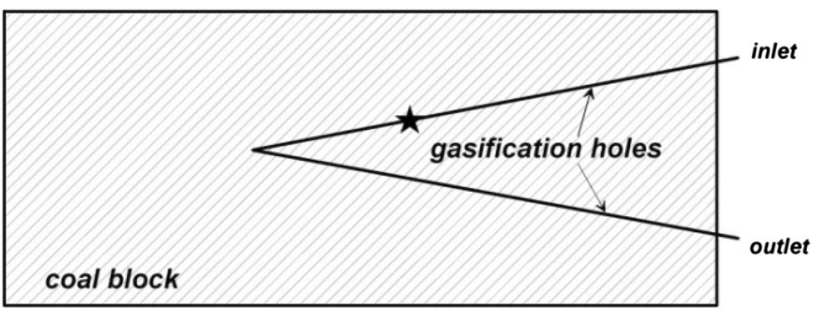

Fig. 4 Schematic horizontal cross-section of coal specimen with layout of gasification holes (plan view). The point where the coal was ignited is marked with a star

The collected data were then analyzed and processed with $3 \mathrm{D}$ algorithms available in RADAN3 program code
(RADAN3 manual 2008). The raw data sets were normalized by a function of gain equalization. The basic operation that had to be then performed on the data was to determine a cut-off threshold for the amplitude of the signals and then to perform a slicing of the cube model. Figure 6 shows spatial views of the surveyed area recorded in both series of measurements without regard to the sign of signal (absolute values).

The images almost fully cover the coal specimen. Only on the top, there may be interference caused by reflection from the sand-coal interface. The locations of the flue channels and the position of the coal ignition point are marked on the images. The cut-off threshold had to be optimized by trials to select the same parameters for both data sets. For that reason 
Fig. 5 A photo views of measurement on "ex situ" model (a) and SIR3000 apparatus with 100 and $1000 \mathrm{MHz}$ transducers (red boxes containing transmitting and receiving radar antennas), used in all experiments described in the paper (b)
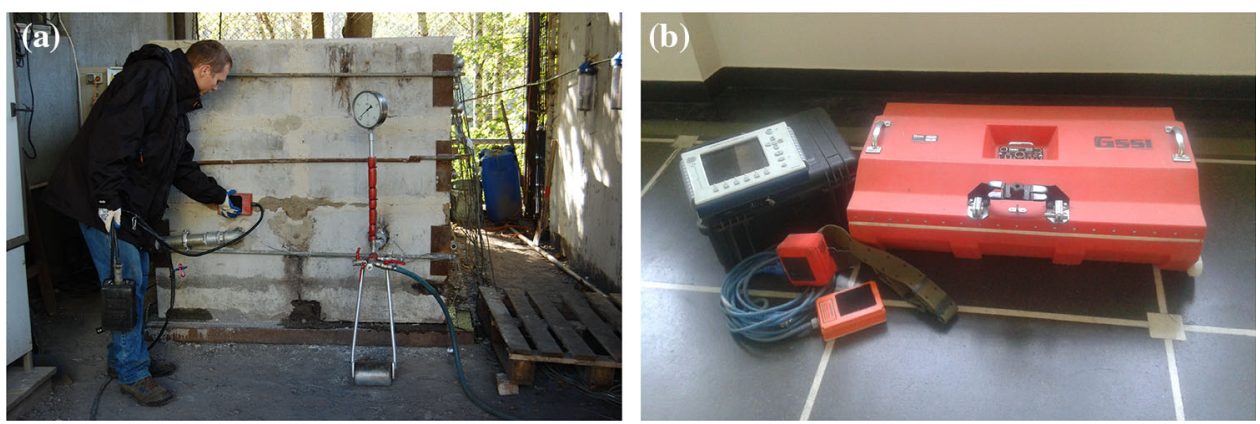

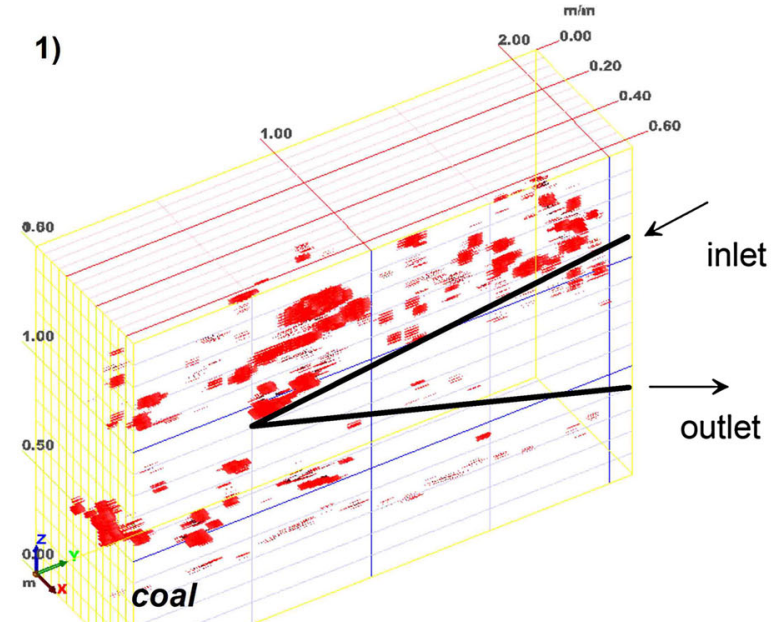

Fig. 6 Spatial views of reflected signals amplitude (absolute values) within coal sample before (1) and after termination of gasification process (2). New regions in coal block reflecting the radar waves

also, reflections can be seen on the image recorded before gasification of coal. These probably come from local heterogeneities in the specimen and should be treated as random or coherent noise. Comparing the images, we can contour six new anomaly regions (a, b, c, d, e, f-in Fig. 6.2). Regions $\mathrm{a}, \mathrm{b}, \mathrm{c}$ and $\mathrm{e}$ are situated near the inlet channel. These anomalies can be interpreted as zones of carbonized coal with voids (located near the ignition point).

Region $\mathrm{d}$ is situated in the vicinity of the outlet hole. The anomaly appears at the region where no signals were recorded before commencing the process. This anomaly should be interpreted as a void or a region of carbonized coal at the crossing of channels, where the flow of gaseous products slows down, which probably leads to water vapour condensation. So it is also possible that in postgasification voids, water in liquid state or other gasification products, such as tar or fly ashes, will be deposited. If the water gathers here, it has relatively the greatest impact on the energy of the reflected signals (Table 2). Anomaly $f$ is located relatively far away from gasification holes. It can correspond to a fissured zone or a region of carbonized coal. It should be emphasized that highlighted regions are

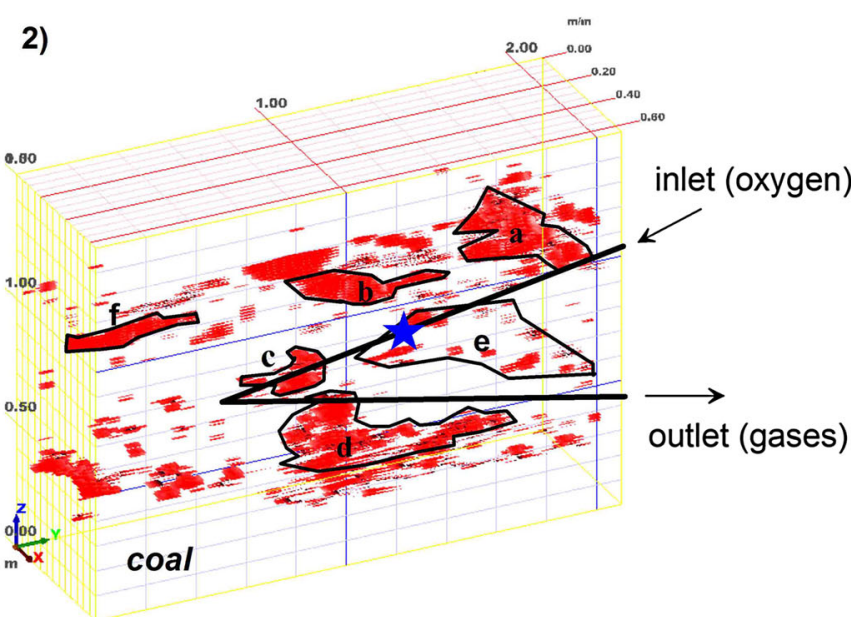

which can be related to voids and char are marked by a, b, c, d, e, and f. Blue star indicates the point of coal inflammation

spatially scattered in coal block and do not form a continuous structure on images constructed using only amplitude as a criterion in data processing. It should be noted also that inlet and outlet holes could not be identified clearly as reflectors of radar signals.

\section{GPR survey at the site of underground gasification experiment}

The coal gasification can be performed both on the surface and in underground conditions. In the first case, coal has to be excavated in mine and brought to a surface plant. There, the coal can be gasified in the reactors of different type, such as fixed bed, fluidized bed or entrained flow. In the second case, the coal is gasified directly in the coal seam (deposit) using boreholes. The boreholes can be drilled from the terrain surface (vertical holes) or from faces of underground mine openings (horizontal holes). In all cases, the gasification process begins with the ignition of coal and supply of oxygen to combustion zone in pure or bound form. This starts with a reaction of coal oxidation in which 
there are produced gases containing carbon: carbon monoxide $(\mathrm{CO})$ and carbon dioxide $\left(\mathrm{CO}_{2}\right)$. The reaction is exothermic and depending on the temperature a certain amount of energy is released in a form of heat. The pressure of gas mixture containing carbon oxides on solid coal forces their movement and other chemical reactions on the travel path called a gasification channel. In this phase called a reduction stage, the coal reacts with carbon oxides and steam which is created from water filling the empty spaces (pores, fissures, and voids between contacting coal cobbles or grains) and evaporating due to temperature increase. Again the carbon oxide $(\mathrm{CO})$ is produced as a result of reaction between coal and carbon dioxide. At these stage also, hydrogen $\left(\mathrm{H}_{2}\right)$ is produced as a product of water decomposition. Both reactions are endothermic. The only exothermic reaction in reduction stage is a coal hydrogenation in which a methane $\left(\mathrm{CH}_{4}\right)$ is produced (Stańczyk et al. 2010).

For the underground coal gasification experiment, an abandoned, shallow hard coal deposit with existing mining infrastructure was chosen. Such conditions exist in the experimental Mine Barbara, which is part of the Central Mining Institute in Poland. Two gasification tests using horizontal holes were conducted in mine Barbara (Wiatowski et al. 2015). Both tests were performed in a $1.5-\mathrm{m}$ thick coal seam at the depth ranging from 15 to $17 \mathrm{~m}$.

The UCG installation for in-seam experiment in Barbara Mine consisted of both surface and underground parts. The installation consisted of the following components: an oxidant supply system, a georeactor, a cooler, an underground and surface dewatering system, a gas collection system and a monitoring system. The reactor was separated by the concrete walls of the mine galleries in the area of ventilation galleries. A horizontal V-shaped fire channel, consisting of two boreholes of $0.14 \mathrm{~m}$ in diameter and $2 \times 17.3 \mathrm{~m}$ in length, was drilled into the coal seam. The coal seam was ignited by a special pyrotechnic charge at a distance of $10 \mathrm{~m}$ from the georeactor inlet. The gasification was initiated by heating the coal seam with oxygen supplied at a flow rate of $10 \mathrm{Nm}^{3} / \mathrm{h}$.

The detailed geology of the site is known from a test borehole B1 drilled some months after the gasification experiment. Carboniferous strata are covered there with a thin layer of Quaternary sediments of 1-2 $\mathrm{m}$ thickness. They have regular quasi-horizontal stratification and appear in the form of beds of sandstones, mudstones and claystones. Between those layers, numerous coal seams of variable thickness, between a few centimeters and a few metres, occur.

The shallowest coal seam at the site, no. 310, lies approx. $16 \mathrm{~m}$ below ground level. It is between 1.3 and $2.0 \mathrm{~m}$ thick. Water appears periodically in the near-surface quaternary sands. The carboniferous strata are not saturated.
According to the mining plan, the seam is oriented almost horizontally and is not faulted. Seam 310 is accessible with two shafts and is cut with numerous roadways. At present, these are used for research purposes. The part of the seam where the gasification experiment was conducted is limited by roadways with a leak-tight concrete lining (Fig. 7).

In a chosen panel of coal, a set of two horizontal holes were bored. The pipes were attached to their outlets and brought out to the surface installation. After point ignition of the coal in one of the holes, oxygen was injected into the coal through this hole. The second hole collected gasification products due to the applied pressure difference between the inlet and outlet (sucking). The gasification experiment was planned to last 3 weeks. Therefore, it was planned to perform 10-12 series of GPR measurements in 2-3 day periods. In fact, the coal gasification process in the seam lasted only $142 \mathrm{~h}$ (from August 1 to August 8 2013). Through the initial $101 \mathrm{~h}$, the process was stable and the composition of the flue gases was stable too. Then, as a result of leakages, there was a gradual decrease in the quality of the flue gases in the georeactor, i.e., a much higher concentration of nitrogen in the flue gases and a lower concentration of hydrogen and carbon monoxide. At this stage, pumping of gaseous nitrogen through the borehole which was used for oxygen supply commenced.

The operation lasted for approximately 4 weeks with breaks for underground inspections, and finally resulted in cooling of the site and extinguishing the oxidation in the coal.

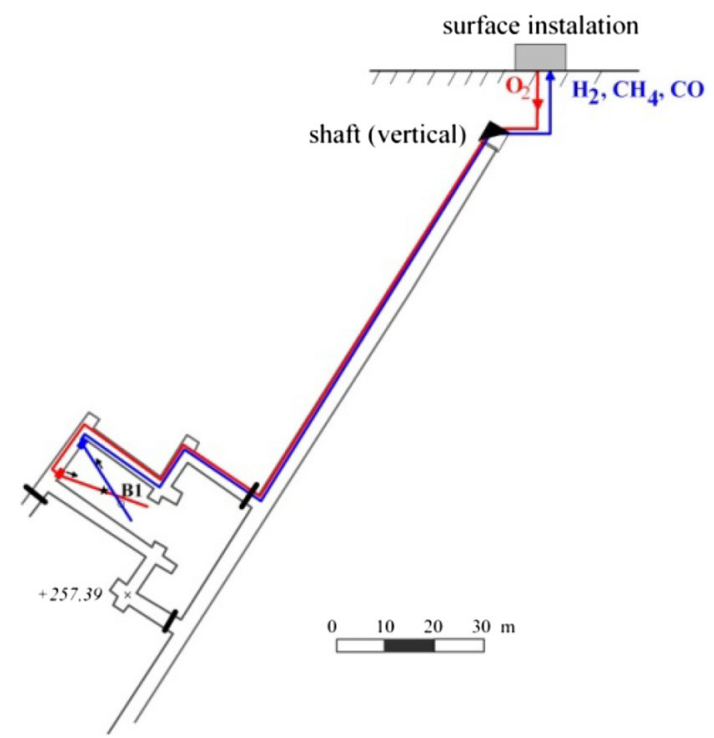

Fig. 7 Layout of flue channels on a plan of roadways in coal seam 310 and test borehole B1 location. The star marks the point of fire setting. The point marked as a vertical shaft is the location where the inlet and outlet holes go underground and then progress horizontally in coal seam toward gasification area 


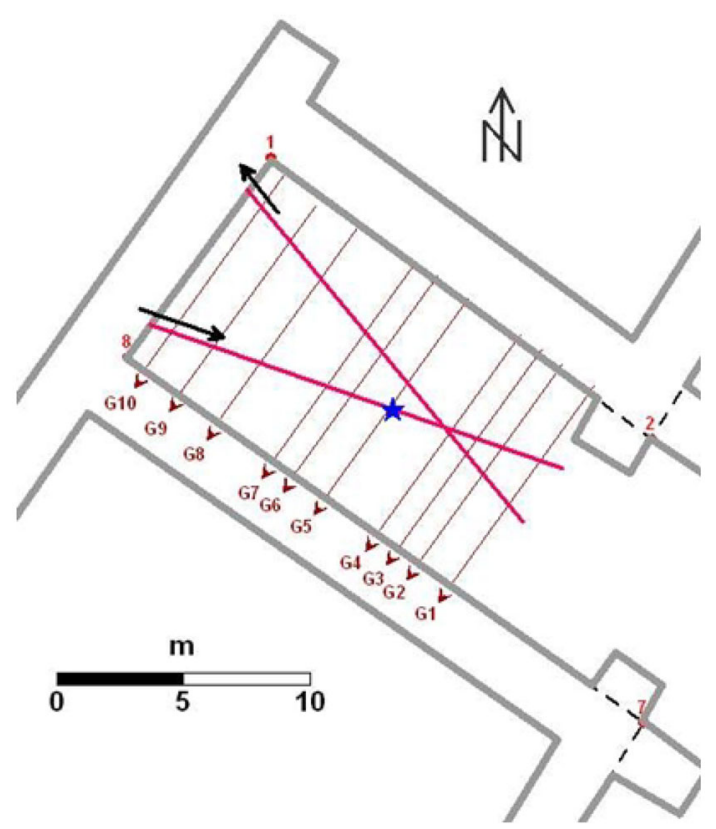

Fig. 8 Layout of GPR profiles on a map of roadways and gasification boreholes in coal seam 310. Arrows show direction of gases flow (in and out)

At the surface over the coal panel selected for the gasification experiment, a set of ten parallel profiles was traced (G1-G10 in Fig. 8). Their length ranged from 8.4 to $9 \mathrm{~m}$. Given the presence of trees and bushes, they were located in places that allowed straight lines to be traced. Therefore, the spacing between profiles was not equal and ranged from 1.3 to $2 \mathrm{~m}$ (Fig. 9). The radar measurements were performed in four time series. The first was done before starting of the gasification process. The second series of measurements was performed after approx. $120 \mathrm{~h}$ from the process start, at the moment when the work of the georeactor became unstable (decrease of flue gases quality). The third series of measurements was performed during cooling of the coal with nitrogen injection (instead of oxygen). The fourth series of measurements was performed after completion of extinguishing works. All measurements were performed with an SIR3000 radar system equipped with $100 \mathrm{MHz}$ mono static transducer (zero-offset between transmitter and receiver). As a result of the measurements, four sets of data were collected.

Datasets from the first and third series of measurements are uniform structurally because they were performed with the use of an odometer. This allowed a constant metric density of traces (20 scans/meter) to be maintained on each profile. Datasets from the second and fourth series of measurements are not so well calibrated metrically due to technical problems with the odometer encountered during the field works. They were calibrated at the processing stage. All the datasets were processed using RADAN3

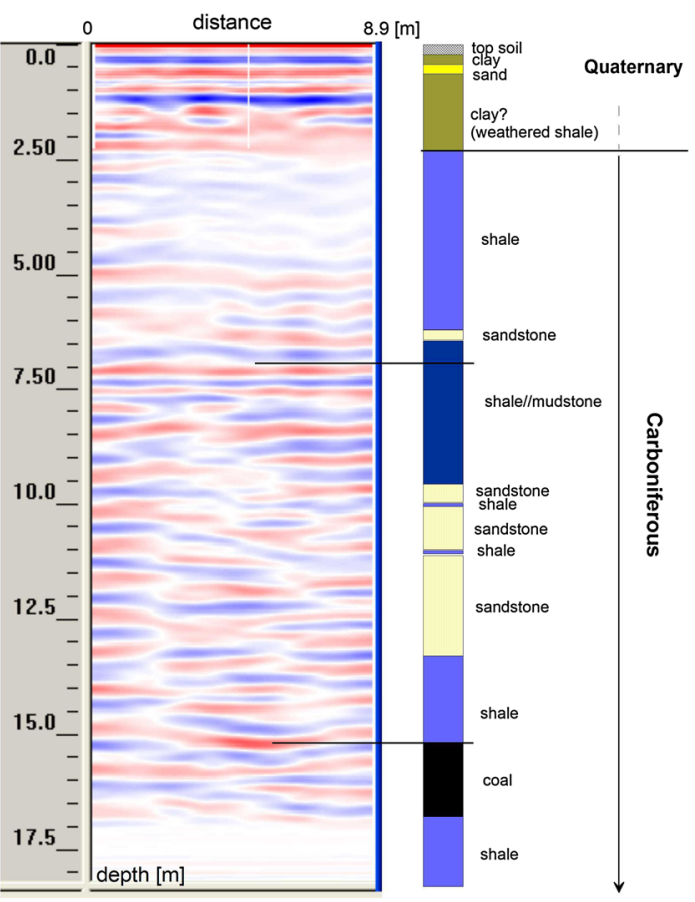

Fig. 9 Radar depth section of profile G3 obtained in third series of measurements and geological log of borehole KDB1 (Kubica 2013)

code for 3D visualization and analysis. This code was applied also for advanced data analyzing using such functions as finite duration impulse response (FIR) in frequency range $0-150 \mathrm{MHz}$ and spatial filtering (2D spatial FFT). For assessment of the radar signals penetration range, we used a function "Max Depth Estimator".

The velocity of radar pulses propagating from the terrain surface to the top of the coal stratum (effective velocity) was determined by a method of diffraction hyperbola fitting combined with multiple migration trials (RADAN3 Manual 2008). As a result of the trials, a velocity value of $V_{\mathrm{ef}}=$ $0.866 \times 10^{5} \mathrm{~km} / \mathrm{s}\left(\varepsilon_{\mathrm{ef}}=12\right)$ was selected for time to depth conversion. The sections calibrated in this way correlate well with the stratification of lithological beds determined in the test borehole B1 $\log$ (Fig. 9), in particular with the mudstone bed top. Quaternary sediments and underlying shale stratum are seen in the image as a layer low attenuating radar signals. Down from the top of the mudstone bed, no specific features are visible. Although it is theoretically possible, the delineation of reflective horizons that could be tied with lithological interfaces is a challenge even for an experienced interpreter of 2D radar data. However, on the raw data image the strongest reflection is visible at a depth correlating well with the top of the coal bed. This feature was used to determine a 2-m thick region (slice) for detailed analysis with the 3D methods available in the RADAN3 computer program. The middle of the slice was selected at a depth of $16.0 \mathrm{~m}$ (half of the coal seam). 


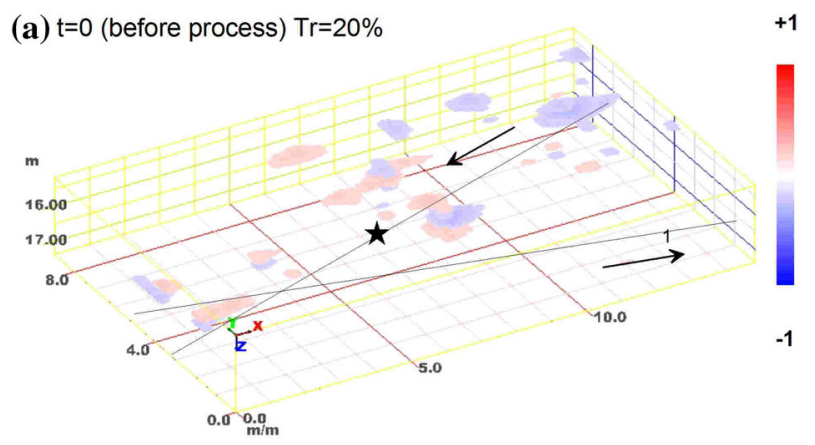

(c) $\mathrm{t}=192 \mathrm{~h}$ (nitrogen injection) $\mathrm{Tr}=20 \%$

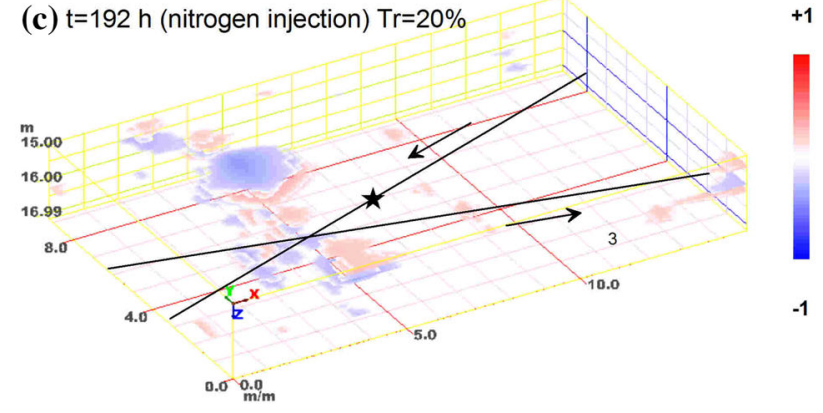

Fig. 10 Time lapse spatial views of reflected signals amplitude exceeding specified thresholds within a slice of the coal seam (width $2 \mathrm{~m}$ ) before gasification process (a), during unstable work of georeactor (b), during cooling of the coal with nitrogen injection

Spatial projections of the values of signal amplitudes within the slice in each measurement series are shown in Fig. 10. The images presented in Fig. 10a, c, and d were obtained removing the portions of data with lowest amplitudes (transparency range of 20\%). Only for the dataset from the second series of measurements, another threshold was used to get a picture which could be compared with the others. For this dataset, the threshold was raised to $50 \%$. In the dataset from the third series of measurements, we see a separated anomalous region located in the vicinity of the crossing of the flue holes. Initially, that anomaly region was interpreted as a void. This image was recorded during the injection of nitrogen into the gasification zone. It significantly differs from images recorded at other stages of the process. No reflections are seen from the regions located in the vicinity of the inlet borehole (clearly visible on Fig. 10b. d). Earlier, through this borehole oxygen was supplied to the coal. If we cut a $3 \mathrm{D}$ model with a very narrow slice $(30 \mathrm{~cm})$ in the middle of coal seam, we get a picture shown in Fig. 11 which corresponds well with the raw data (Fig. 9). Interpretation of the anomaly seen on Figs. 10c and 11 was positively verified by gravity survey (Kotyrba et al. 2015) and a test borehole B1 drilled within the anomaly area.

When the drilling reached the top of the coal bed, the coring head dropped by $15 \mathrm{~cm}$. This was followed by the (b) $\mathrm{t}=120 \mathrm{~h}$ (unstable process) $\mathrm{Tr}=50 \%$

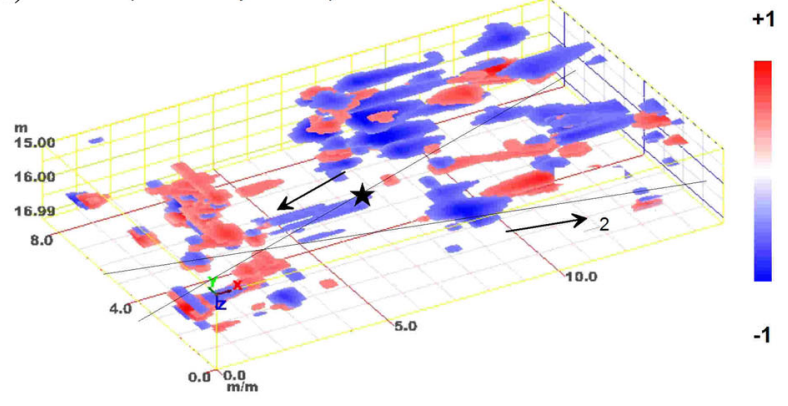

(d) $\mathrm{t}=288 \mathrm{~h}$ (after process) $\mathrm{Tr}=20 \%$

$+1$

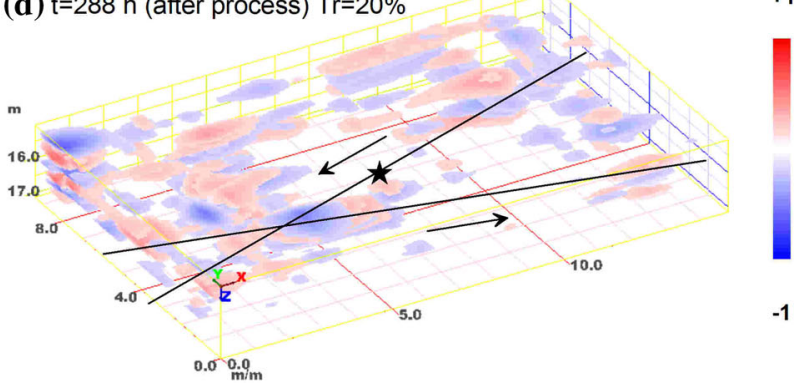

(c), and after extinguishing the fire in the coal (d). The star marks the point of coal inflammation. Arrows mark the direction of gases flow. Scale for amplitude and phase of signals on vertical bar

disappearance of the drilling fluid. Doubts arose after analysis of the fourth series of data (Fig. 10d). On this image also, a space of relatively high amplitudes is visible in the anomaly region. However, the area is smaller. The third series of measurements was conducted during cooling of the coal bed. This could cause vapour condensation and water release in fissures (voids) created by coal gasification. When the cooling of the coal was terminated, the water could move out of these fissures and flow to other ones or flow out the panel. Because the shape of the gasification zone and the development of char and cavity surfaces are crucial for process control, the data from the third series of measurements were used for simulation of the coal gasification progress in time. The amplitudes of signals were magnified (multiplied by a constant valuerange gain function). The resulting plan view of the slice from Fig. 10c is shown in Fig. 11.

On this image, a contour of the zone containing carbonized coal (char) and voids is marked. The contour line was determined by complex analysis of the radar data, direct in-seam observations and test boreholes. Therefore, it does not include all the regions determined automatically by the applied procedures of radar data processing. On the image, the most probable scenario of process development is shown by black arrows. According to that, after the moment of coal inflammation, the gasification surface was developing near the 


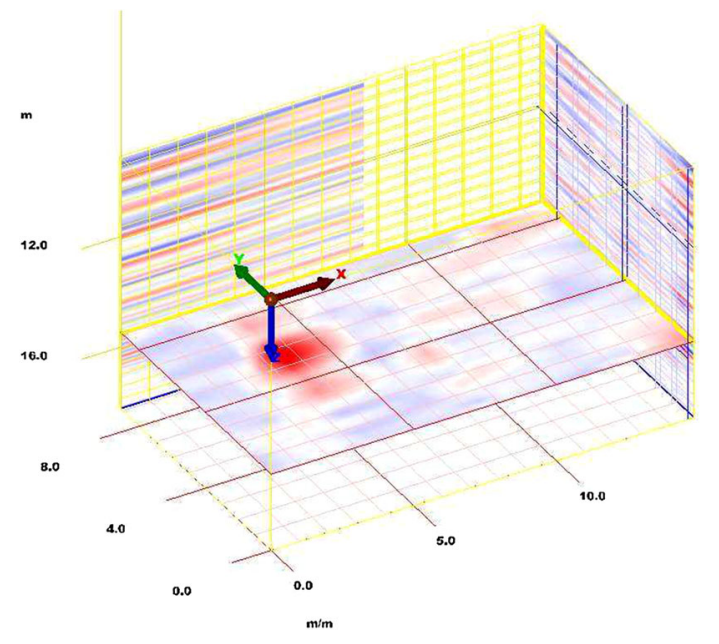

Fig. 11 A spatial view of radar signal amplitudes in the middle of coal seam (data from third series of measurements)

crossing of the boreholes. This was probably during the stable stage of georeactor work. Afterwards, the outlet hole was blocked by tar or ash and the gasification surface began to move back to inlet of the oxygen supplying hole.

On the line of the installation's outlet hole, two regions which may correspond to blocking objects are visible. They are indicated by blue arrows. The rest of the regions visible on the image presented in Fig. 12 can be treated as artefacts. They are located near the edges of the coal panel and do not link with gasification paths.

\section{Discussion}

The main objective of the research described in the paper was to analyze the possibility of using time lapse data from GPR measurements to image the zone of coal gasification. Theoretical considerations indicate that interfaces created in coal during gasification are characterized by low contrast of dielectric permittivity values. The reflectivity of electromagnetic waves on these interfaces depends relatively weakly on the temperature. At the beginning of the process, when the temperature of the coal is low, the reflection coefficient for these interfaces is the highest. It decreases with rising temperature. After process termination, when the temperature returns to its initial state, the values of the reflection coefficients also return to values close to the initial ones. The important finding from this research concerns issues connected with the attenuation of electromagnetic waves in gasified coal. This parameter significantly drops in the gasification zone following the temperature rise. As a result, the penetration range of electromagnetic pulses significantly increases. This feature can be used by "in-seam" techniques of gasification zone sensing.

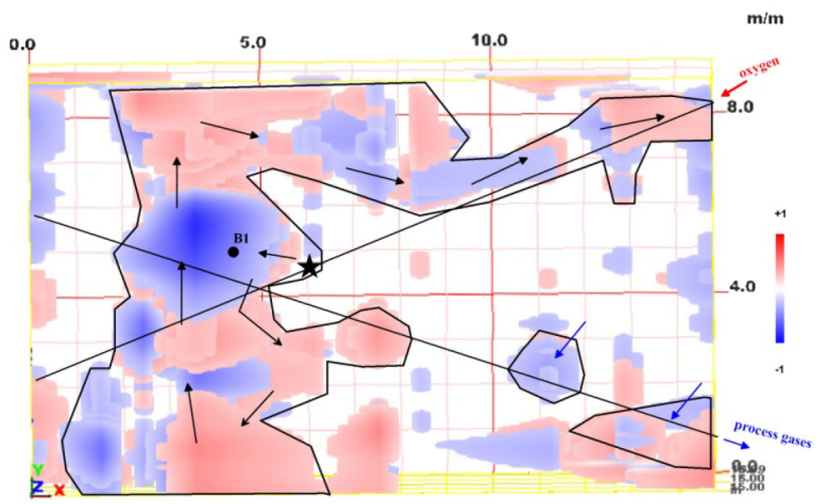

Fig. 12 Layout of GPR profiles on a map of roadways and gasification boreholes in coal seam 310. Arrows show direction of gases flow (in and out). Enclosed regions show the areas in coal seam transformed by gasification process

As shown by the analysis of reflection coefficients, the small difference between the dielectric constant values of carbonized coal (char) and gases in the gasification void indicates that in 3D data processing methods based only on the amplitude criteria, determination of threshold level in transparency range function is a very subtle work. In some cases, there will be only a small difference in dielectric properties on the char-void interface. In those cases, it can be impossible to find the interface in the dataset at all. Nevertheless, the coal-char interface in all analyzed cases always has proper reflectivity. This interface determines the contour of the gasification zone (the part of the coal seam involved in the process).

For datasets collected in the field experiment, the threshold value for transparency in RADAN3 code (the visible percentage of the slice dataset) for images presented in this paper was set to $T_{\mathrm{r}}=20 \%$. The resolution parameter in range slicing was set to a value of 32 . Only for the image recorded during the gasification process, we had to use a much higher transparency threshold (Fig. 10b) to obtain an image comparable with others. The amplitudes of signals reflected from gasification interfaces in these datasets increased by a factor of 2.5. This can be attributed to dielectric permittivity change due to the flow of gases that were created in the process. For the gasification process characterization, only the images obtained in the second, third, and fourth series of measurements are of value. They show that the process developed mainly in the vicinity of the hole supplying oxygen and a region located near the crossing of the holes. The regions characterized by relatively high amplitude of reflected signals can be interpreted as a carbonized coal (char). Within them are probably voids and fissures. Their contours, except for the case presented in Fig. 10c, are difficult to determine. It should be pointed out that after the process termination, an outcrop was made at the inlet of the oxygen supplying 
borehole. This revealed a tubular structure containing void and carbonized coal of approximate height $0.80-1.0 \mathrm{~m}$ and a length of several metres in the coal seam. This structure corresponds well to the image simulating the gasification progress drawn on the basis of the amplitude of the reflected signals distribution (Fig. 12).

Tests performed in the laboratory and "in situ" conditions showed that the method can provide valuable data for assessing and monitoring gasification surfaces in the UCG processes. The advantage of the GPR method is its high resolution and the possibility of determining the spatial shape of various zones and forms created in the coal by the gasification process. The disadvantage of the method is the strong dependence of signals reflection amplitude on water content (in a liquid state). Therefore, the criterion of maximum amplitude used for data interpretation can lead to the positioning of regions in the coal seam containing water in a liquid state (for instance, water-filled fissures). The presence of free water in a geological medium is dependent on its temperature. In described gasification experiment, water could exist in a liquid state up to a temperature of approx. $120-150{ }^{\circ} \mathrm{C}$. This factor seems to be important particularly in real geological environments where the natural water or water created in the gasification process can exist in gaseous and vapour states in parallel.

\section{Conclusions}

Described in the article, results of theoretical considerations and tests performed in "ex situ" and "in situ" conditions indicate that in certain cases the GPR method may be useful for sensing the coal gasification process while in others it does not. In experiments, we used a standard, commercial radar system for measurements carried out in reflective mode (designed for geotechnical purposes) manufactured by Geophysical Survey Systems Inc. (GSSI). The research results obtained during tests on models 'ex situ' do not doubt. For tests conducted 'in situ', over an area where the coal seam was gasified underground, there are doubts concerning the depth range of radar mapping with use of the GSSI $100 \mathrm{MHz}$ antennae in mono static mode. This points to the need to use or design special radar systems (with a much higher power of signal transmitter and other antenna types) for "in situ" method application in subsequent experiments.

Acknowledgments The results presented in this work have been obtained within the framework of HUGE2 (Hydrogen Oriented Underground Coal Gasification for Europe) - a European research Project co-financed by the Research Fund for Coal and Steel under contract no. RFCR-CT-2011-00002 and the Polish Ministry of Science and Higher Education.

\section{Compliance with ethical standards}

Conflict of interest On behalf of all authors, the corresponding author states that there is no conflict of interest.

Open Access This article is distributed under the terms of the Creative Commons Attribution 4.0 International License (http://crea tivecommons.org/licenses/by/4.0/), which permits unrestricted use, distribution, and reproduction in any medium, provided you give appropriate credit to the original author(s) and the source, provide a link to the Creative Commons license, and indicate if changes were made.

\section{References}

Annan AP (2001) Ground penetrating radar. Workshop Notes. Sensor \& Software Inc., Mississauga

Bhutto AW, Bazmi AA, Zahedi G (2013) Underground coal gasification. From fundamentals to applications. Prog Energy Combust Sci 39:189-214. https://doi.org/10.1016/j.pecs.2012. 09.004

Crawford FS (1968) Waves. Mc Graw-Hill, New York

Ebbing D, Gammon DS (2011) General Chemistry-Ninth Edition, 9th edn. Cengage Learning, Brooks/Cole

Gaffney JS, Marley NA (2009) The impacts of combustion emissions on air quality and climate-from coal to biofuels and beyond. Atmos Environ 43:23-36. https://doi.org/10.1016/j.atmosenv. 2008.09.016

Gut Z, Niedziela W, Szymczyk J (2004) Electrical capacitance tomography electrical aspects of combustion. J KONES Intern Combust Engines 11:212-217 (e-ISSN:2354-0133)

Hanninen P, Hanninen P, Kopponen L, Koskiahde A, Maijala P, Pollari R, Saarenketo T, Sutinen R (1992) Ground penetrating radar. The Finnish Geotechnical Society-The Finnish Buiding Centre Ltd., Tampere. ISBN 951-662-238-X

Himmelblau DM (1960) Solubility of inert gases in water. $0^{0} \mathrm{C}$. to near critical point of water. J Chem Eng Data 5(1):10-15. https:// doi.org/10.1021/je60005a003

Itakura K, Wakamatsu M, Sato M, Goto T, Yoshida Y, Ohta M, Shimada K, Belov A, Ram G (2009) Fundamental experiments for developing underground coal gasification (UCG) systems. Mem Muroran Inst Tech. 59:51-54 (http://hdl.handle.net/ 10258/448)

Janoszek T, Sygal A, Bukowska M (2013) CFD simulation of temperature variation in carboniferous strata during UCG. J Sust Min 12(4):34-44

Khan MM, Mmbaga JP, Shirazi AS, Liu Q, Gupta R (2015) Modelling underground coal gasification-A review. Energies 8(11):12603-12668

Kostur K, Laciak M, Durdan M, Kacur J, Flegner P (2015) Low calorific gasification of underground coal with a bigger humidity. Measurement 63:69-80

Kotyrba A (1999) Electric conductivity and self-heating ability of hard coal. Arch Mining Sci 44(3):435-447

Kotyrba A, Stańczyk K (2013) Application of a GPR technique for monitoring of simulated underground coal gasification in a large scale model. Near Surf Geophys 11(5):505-515. https://doi.org/ 10.3997/1873-0604.2013030

Kotyrba A, Kortas Ł, Stańczyk K (2015) Imaging the underground coal gasification zone by microgravity surveys. Acta Geophys 63(3):634-651. https://doi.org/10.1515/acgeo-2015-0002

Kubica J (2013) Sprawozdanie z wiercenia otworu badawczego KDB1w rejonie georeaktora w Kopalni Doświadczalnej "Barbara" w 
Mikołowie. Central Mining Institute (unpublished report), Katowice

Marland S, Merchant A, Rowson N (2001) Dielectric properties of coal. Fuel 80:1839-1849. https://doi.org/10.1016/S00162361(01)00050-3

Nourozieh H, Kariznovi M, Chen Z, Abedi J (2010) Simulation study of underground coal gasification in Alberta reservoirs: geological structure and process modeling. Energy Fuels 24(6):3540-3550

RADAN3 Manual (2008) Geophysical Survey Systems. New Hampshire, USA

Raumenergie-Förder Gesellschaft. Dielectric chart (2013) (www. rafoeg.de)

Sarraf Shirazi A, Karimipour S, Gupta R (2013) Numerical simulation and evaluation of cavity growth in in situ coal gasification. Indust Eng Chem Res 52(33):11712-11722

Schmidt JW, Moldover MR (2003) Dielectric permittivity of eight gases measured with cross capacitors. Int $\mathrm{J}$ Thermophys 24(2):76-403

Shindell D, Faluvegi G (2010) The net climate impact of coal-fired power plant emissions. Atmos Chem Phys 10:3247-3260. https://doi.org/10.5194/acp-10-3247-2010
Stańczyk K, Smoliński A, Kapusta K, Świądrowski J, Wiatowski M, Kotyrba A, Rogut J (2010) Dynamic experimental simulation of hydrogen oriented underground gasification of lignite. Fuel 89:3307-3314. https://doi.org/10.1016/j.fuel.2010.03.004

Su F, Nakanowataru T, Itakura K, Ohga K, Deguchi G (2013) Evaluation of structural changes in the coal specimen heating process and UCG model experiment for developing efficient UCG systems. Energies 6(5):2386-2406. https://doi.org/10. 3390/en6052386

Turner G, Yelf R, Hetherly P (1989) Coal mining applications of ground penetrating radar. Exploration Geophysics 20(2):165-168. https://doi.org/10.1071/EG989165

Wiatowski M, Kapusta K, Świądrowski J, Cybulski K, LudwikPardała M, Grabowski J, Stańczyk K (2015) Technological aspects of underground coal gasification in the experimental "Barbara" mine. Fuel 159:454-462. https://doi.org/10.1016/j. fuel.2015.07.001 